\title{
Research on Dynamics Characteristics of the Rotation Axis in CNC Machine Tool
}

\author{
Dong $X^{1} e^{1, a,{ }^{*}}$, zhu jianqu ${ }^{1, b}$, Wang feng ${ }^{1, c}$ \\ ${ }^{1}$ School of Electric \& Information Engineering, Chongqing University of Science and Technology, \\ Chongqing, China \\ axiedongcq@126.com, bzhujianqu@126.com, cwfjqh@126.com \\ ${ }^{*}$ Corresponding author
}

Keywords: CNC Machine Tools, Rotation Axis, Dynamic Characteristics, Contour Error Abstract: The dynamic characteristics of the rotary axis feed system has influence on the contour error. This paper presents a mathematical model of rotary table drive system of the CNC machine tool by using of mechanical dynamics. The model includes the simulation model of AC servo motor and the rotary table drive system. The time domain and frequency domain simulation characteristic curves can be obtained to show the dynamic performance of the rotation axis. By the simulation results, the rotary axis driver system's rapidity and stability can be analyzed. The study results can provide a reference for the investigating the dynamic performance influence on errors.

\section{Introduction}

With the rapid development of the high-feed rate in $\mathrm{CNC}$ machining process, the high-performance servo feed system is required to meet the products machining precision and efficiency. However, there is friction between the moving parts of the drive system, as well as cutting forces between the workpiece and tool. They will cause servo feed system a greater interference and make the following precision become poor. The servo feed system parameters have different effects on the dynamic performance.

In order to analyze errors caused by position tracking errors of feed system. Some researchers proposed that the contour tracking precision of the part relates to the stability and dynamic characteristic of servo drive system by position closed loop control[1,2]. The effectiveness of dynamics of the feed drive is investigated comprising of rigid body motion, friction and ball screw vibrations, and the characteristics of two rotary tables is measured and compared[3,4]. The others established two degrees freedom vibration model to estimate the transfer function of the actual system[5,6]. This paper proposed a rotation axis feed system model to analyze the dynamic characteristics of the feed system.

\section{The Mathematical Model of the Rotation Axis Feed System}

With the application of the rotation axis, the $\mathrm{CNC}$ machine tool save the processing time, improve efficiency, and carry a variety of complex parts machining. The rotation axis uses the different mechanical drive mechanism which the translational axis are used the ball screw and the rotation axis are mostly used worm driver, the rotating axis feed system is modeled in the following.

The angular displacement of rotation servo motor is as input of the mechanical transmission mechanism. The output is the angular displacement of the rotary table or spindle head. The worm drive mechanism is usually used in five-axis CNC machine tools, which can effectively transfer torque and ensure high accuracy. Here, taking the worm gear as an example, the rotary table and the drive mechanism is simplified as the 4 freedom degrees model in Fig. 1.

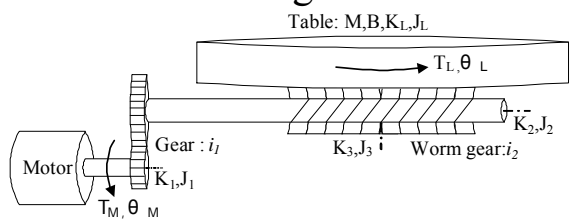

Fig. 1 The rotation axis mechanical transmission construction 
Where, $\theta_{\mathrm{M}}$ and $T_{\mathrm{M}}$ is the input angle and torque of AC servo motor respectively, $\theta_{\mathrm{L}}$ is the output of the rotary table; $i_{1}$ is gear ratio, $i_{2}$ is the worm gear ratio; $K_{1}, J_{1}$ is the equivalent torsional stiffness and moment inertia of the motor shaft; $K_{2}, J_{2}$ is that of the worm shaft; $K_{3}, J_{3}$ is that of rotational table shaft; $\mathrm{k}$ is the equivalent torsional stiffness coefficient; $M$ and $B$ is mass and viscous damping of the $\mathrm{CNC}$ rotary table rotating part respectively.

The dynamic equation is (1).

$$
\left\{\begin{array}{l}
J_{1} i_{1} i_{2} \theta_{L}^{\sigma_{L}}(t)-k\left(\theta_{M}(t)-i_{1} i_{2} \theta_{L}(t)\right)=-\frac{1}{i_{1}} T_{2}(t) \\
J_{2} i_{2} \theta_{L}(t)=T_{2}(t)-\frac{1}{i_{2}} T_{3}(t) \\
\left(J_{3}+J_{L}\right) \theta_{L}^{\delta_{L}}(t)+B \theta_{L}^{\&}(t)=T_{3}(t)
\end{array}\right.
$$

After laplace transform, the system transfer function is:

$$
G(s)=\frac{\theta_{L}(s)}{\theta_{M}(s)}=\frac{K / i_{1} i_{2}}{J_{\Sigma} s^{2}+B_{\Sigma} s+K}
$$

Where: $J_{\Sigma}=J_{1}+\frac{1}{i_{1}^{2}} J_{2}+\frac{1}{i_{1}^{2}} \frac{1}{i_{2}^{2}} J_{3}+\frac{1}{i_{1}^{3}} \frac{1}{i_{2}^{3}} J_{L}, B_{\Sigma}=B_{L} / i_{1} i_{2}$

It is shown that the rotation axis mechanical transmission model can be seen as a second-order link. Let's set $\omega_{\mathrm{n}}$ and $\xi$ as $\omega_{n}=\sqrt{k / J_{\Sigma}}, \xi=B_{\Sigma} K^{2} / 2 \sqrt{k J_{\Sigma}}, \omega_{\mathrm{n}}$ is the undamped natural frequency of the second order link; $J_{\Sigma}$ is moment inertia of CNC table; $\xi$ is damping ratio of the second-order link.

The mathematical model of the three-phase AC permanent magnet synchronous motor involved characteristics of multiple variables, strong coupling and nonlinear, the model is more complex. The most common method used the vector transform for modeling. The linearly decoupling method is used to be similar to the DC motor. The motor in d-q coordinate equation is:

$$
\left\{\begin{array}{l}
u_{s d}=R_{s} i_{s d}+\frac{d \phi_{r d}}{d t}-\omega_{e} \phi_{r q}, \quad u_{s q}=R_{s} i_{s q}+\frac{d \phi_{r q}}{d t}-\omega_{e} \phi_{r d} \\
T=\frac{3}{2} P_{n}\left[\psi_{f} i_{s q}-\left(L_{d}-L_{q}\right) i_{s d} i_{s q}\right]
\end{array}\right.
$$

Where: $u_{s d}, u_{s q}$ is d-q axis stator voltage component; $i_{\text {sd }}, i_{\text {sq }}$ is current component; $\Phi_{r d}, \Phi_{r q}$ is flux component; $L_{\mathrm{d}}, L_{\mathrm{q}}$ is inductance component; $\mathrm{R}_{\mathrm{s}}$ is the stator resistance; $\omega_{e}$ is electric motor angular velocity; $\Psi_{f}$ is rotor permanent magnet flux constant; $P_{n}$ is the number of pole pairs.

The torque current component $i_{s q}$ plays a major role to electromagnetic torque generation. Typically excitation component contributed little to the electromagnetic torque generation. In order to obtain the maximum available torque, usually controlled $i_{s d}=0$, then:

$$
\left\{\begin{array}{l}
u_{s d}=-P_{n} \omega_{e} \Psi_{r q}, \quad u_{s q}=R_{s} i_{s q}+L_{q} \frac{d i_{s q}}{d t}+P_{n} \omega_{e} \Psi_{f} \\
T=\frac{3}{2} P_{n} \Psi_{f} i_{s q}
\end{array}\right.
$$

It can be seen that the torque is proportional only to the armature current $i_{s q}$. In case of $i_{s d} \equiv 0$, the current component $i_{s d}$, $i_{s q}$ get a good decoupling control in the d-q coordinate. So the mathematical model is similar to the excited DC motor linearized model. The $u_{s d}$ component can produce torque armature made the torque have nonlinear characteristic. But in the actual, due to $L_{q} i_{s q} \leq \Psi_{f}$, so the influence of the armature reaction is negligible. The $u_{s q}$ and the induction electromotive force $E_{f}$ is:

$$
\begin{aligned}
& u_{s q}=R_{s} i_{s q}+L_{q} \frac{d i_{s q}}{d t}+P_{n} \omega_{e} \psi_{f} \\
& E_{f}=P_{n} \omega_{e} \psi_{f}=K_{e} \omega_{e}
\end{aligned}
$$

Where $K_{\mathrm{e}}=P_{\mathrm{n}} \Psi_{\mathrm{f}}$ is coefficient of induction electromotive force, so from (5) and (6), the voltage and current transfer function can be obtained by Laplace transform.

$$
\frac{I_{s q}(s)}{U_{s q}(s)-E_{f}(s)}=\frac{1}{L_{q} s+R_{s}}
$$

The three-phase AC permanent magnet synchronous motor mechanical equation is: 


$$
T-T_{L}=J \frac{d \omega_{e}}{d t}
$$

By Laplace transform, it can be obtained:

$$
\frac{T(s)-T_{L}(s)}{\omega_{e}(s)}=J \mathrm{~s}
$$

According to equation (2) and (9) can be established a simulink block diagram for AC servo motor drive system of CNC rotary table, it is shown in Fig. 2.

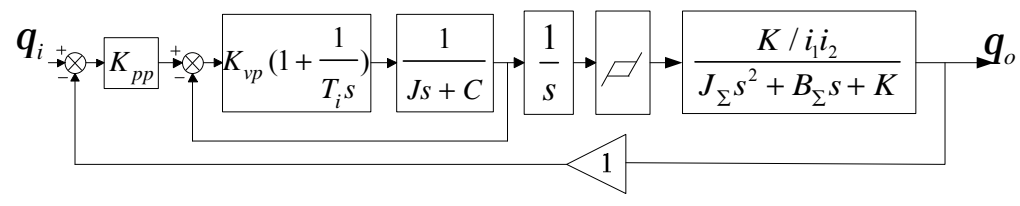

Fig. 2 The block diagram of the feed system

\section{Simulation of Dynamic Characteristics}

From Fig. 2, it can be known that the AC servo drives system include the speed loop and the position control loop. A PI controller is selected in the speed loop, which the tuning parameters can ensure the motor rotation angle smoothness and accurately. For the position loop, the PID controller is selected, which the tuning parameters can ensure the accuracy and rapidity of the motor output.

During the simulation, the moment inertia is taken the motor shaft $J_{1}=110 \mathrm{~kg} \cdot \mathrm{m}^{2}$. Similarly, $J_{2}=220 \mathrm{~kg} \cdot \mathrm{m}^{2}$, and $J_{3}=3800 \mathrm{~kg} \cdot \mathrm{m}^{2}$. The CNC rotary table mass is $300 \mathrm{~kg}$, the gear ratio $Z_{1}: Z_{2}=1: 4$, the worm gear ratio $Z_{3}: Z_{4}=1: 180$. The second-order system undamped natural frequency is calculated from the known parameters.

The system simulation curves for different values of $\xi$ are shown in Fig. 3. As can be seen from these simulation curve, when the natural frequency of the system $\omega_{\mathrm{n}}$ remain unchanged, the fast response of the $\mathrm{CNC}$ rotary table has increased with the decrease of the damping ratio $\xi$, but the relative stability of system has reduced. Therefore, If you require CNC rotary table has better stability and fast, the damping ratio $\xi$ should be select roughly in the range of $0.6 \sim 0.8$.

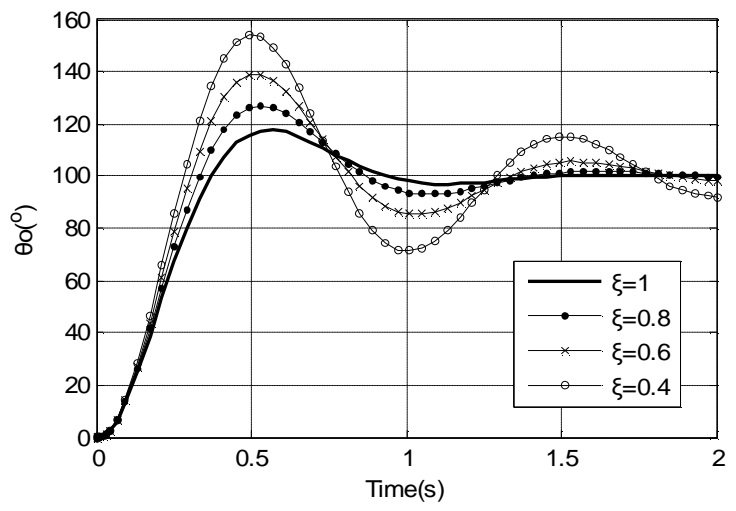

Fig. 3 The system simulation curves of the different values of $\xi$

The damping ratio $\xi$ is proportional to transmission coefficient $\mathrm{K}$ and viscous damping $\mathrm{B}_{\mathrm{L}}$ of $\mathrm{CNC}$ rotary table, and inversely proportional to the square root of the moment of inertia the equivalent $J_{\Sigma}$ and equivalent stiffness coefficient $\mathrm{k}$. It is shown that the rotary table drive system should be increased $\mathrm{k}$, decreased $\mathrm{J}_{\Sigma}$ ensure the stability and fast of the $\mathrm{NC}$ rotary table.

The Nyquist stability criterion is a identification method on the stability analysis in frequency domain, which is using the system's open-loop frequency characteristics to obtain the stability of the closed loop system. It is proposed to improve the control system performance and stability. The open-loop transfer function of the CNC rotary table drive system is as (10).

$$
G(s)=\frac{225}{s^{2}+24 s+225}=\frac{225}{(s-12-9 j)(s-12+9 j)}
$$

The open-loop transfer function of the Nyquist graphs is shown in Fig. 4. It can be seen that the amplitude and phase frequency characteristic curve is not surrounded by $(-1,0 \mathrm{j})$ point. The system 
has been proved to be stable. The system bode graphs is as Fig. 5 .

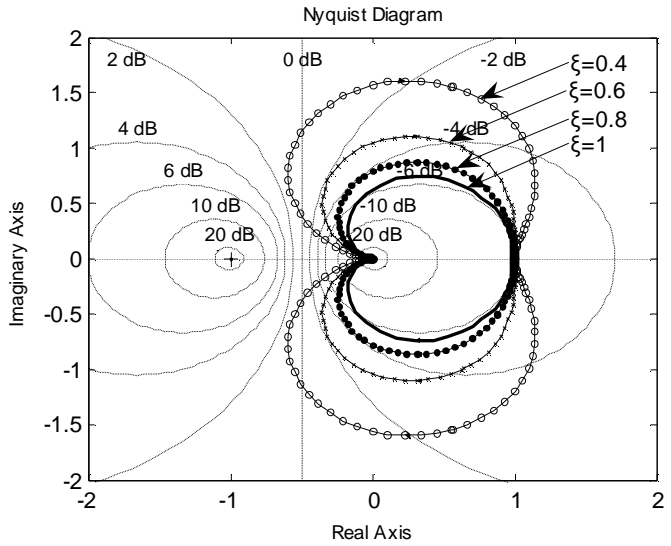

Fig. 4 The system Nyquist graphs

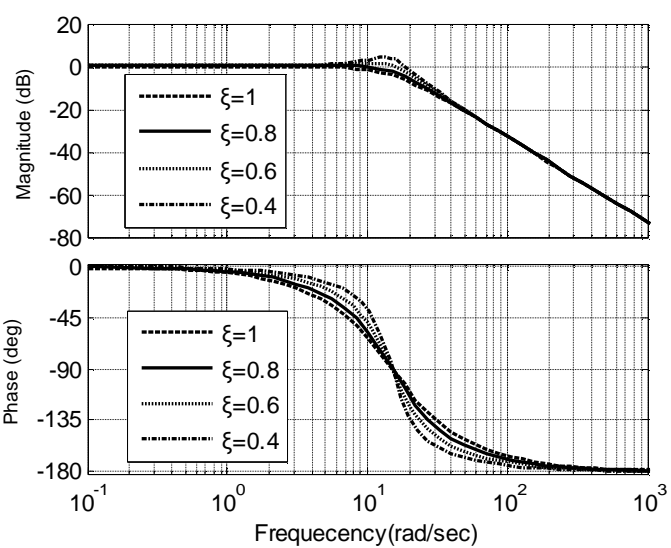

Fig. 5 The system bode graphs

Although the PID controller is selected for the AC servo motor position control loop system, which can only guarantee the accuracy of the motor output to a certain extent the corner, but not eliminate the errors generated by the gear and worm gear gap. The presence of worm drive gear clearance will affect the output of CNC turret angle $\theta_{\mathrm{L}}$ error and the transmission accuracy. Therefore, in order to improve the accuracy of the transmission system, at first, it is necessary to improve the precision gears, minimize installation error. And the second, the CNC rotary table detection devices will be mounted to constitute semi-closed or closed loop servo system to improve the accuracy of CNC rotary table output angle. The third is to set suitable numerical control system compensation parameters to reduce the impact on the drive precision.

\section{Conclusion}

By modeling the rotation axis servo driver system of CNC machine tools, the time domain and frequency domain simulation characteristic curves can be obtained to show the dynamic performance of the rotation axis. By the simulation results, the rotary axis driver system's rapidity and stability can be analyzed. The results can provide a role base to improve the rotation axis moving precise by the adjustment dynamic performance parameters of the $\mathrm{CNC}$ machine tool.

\section{Acknowledgment}

This research is funded by Research Foundation of Chongqing University of Science \& Technology, the project No. is CK2015Z30.

\section{References}

[1]Y. Keron. Variable-gain cross-coupled controller for contouring, Annals of CIPR, 104(1991) 371-374.

[2]K. Erkorkmaz, Y. Altintas. High speed CNC system design. Part $\square$ : modeling and identification of feed drivers. Int. J. of Machine tools \& Manufacture, 41 (2001) 1487-1509.

[3]K.M.M. Dassanayake, M. Tsutsumi, R. Sato, et al. Motion Characteristics of High Performance Rotary Tables for CNC Machines. Proceedings of IMECE2008. 11 (2008) 27-36

[4]R. Sato, M. Tsutsumi. Modeling and controller tuning techniques for feed drive systems. Proceedings of IMECE2005, Florida USA, 11 (2005) 669-679

[5]G.T.C. Chiu, M. Tomizuka. Contouring control of machine tool feed drive systems: a task coordinate frame approach. Control Systems Technology on IEEE Transactions, 9 (2001) 130-139.

[6]X. Huang, L. Shi. Simulation on a fuzzy-pid position controller of the CNC servo system, in Intelligent Systems Design and Applications 2006 on IEEE, 1 (2006) 305-309. 